Revue

Revue de l'histoire des religions

del'histoire des religions

\title{
Les Sages du Talmud et l'Évangile selon Matthieu
}

Dans quelle mesure l'Évangile selon Matthieu était-il connu des Tannaïm?

The Sages of the Talmud and the Gospel according to Matthew. To what extent was the Gospel according to Matthew known to the Tannaim?

Dan Jaffé

\section{CpenEdition}

Journals

Édition électronique

URL : http://journals.openedition.org/rhr/7544

DOI : $10.4000 /$ rhr.7544

ISSN : 2105-2573

Éditeur

Armand Colin

Édition imprimée

Date de publication : 1 décembre 2009

Pagination : 583-611

ISBN : 978-2200-92592-5

ISSN : 0035-1423

Référence électronique

Dan Jaffé, «Les Sages du Talmud et l'Évangile selon Matthieu », Revue de l'histoire des religions [En ligne], 4 | 2009, mis en ligne le 01 décembre 2012, consulté le 10 décembre 2020. URL : http:// journals.openedition.org/rhr/7544; DOI : https://doi.org/10.4000/rhr.7544 


\title{
Les Sages du Talmud et l'Évangile selon Matthieu Dans quelle mesure l'Évangile selon Matthieu était-il connu des Tannaïm?
}

\begin{abstract}
Cet article propose une interprétation du terme " guilyonim » tel qu'il apparaît dans la littérature talmudique. À l'issue d'une étude philologique et historique, il est suggéré d'y lire le terme "Évangile " comme une translittération du grec à l'hébreu. Dans le Talmud de Babylone, en Sabbath 116a-b, un verset de l'Évangile selon Matthieu est cité avec des distorsions. En prenant en considération les attestations patristiques relatives à l'origine hébrä̈que de l'Évangile selon Matthieu, il est possible de supposer qu'un certain nombre de tannaïm, à partir du début du $I I^{e}$ siècle, connaissaient des fragments de cet évangile dans ses versions hébraïques (ou araméennes). Si cette hypothèse est retenue, il devient possible de comprendre la vive polémique de R. Gamaliel à l'encontre de certains versets de l'Évangile selon Matthieu et contre les judéochrétiens en général.
\end{abstract}

The Sages of the Talmud and the Gospel according to Matthew. To what extent was the Gospel according to Matthew known to the Tannaim?

This article presents an interpretation of the term "guilyonim " that appears in the Talmudic literature. In the wake of historical and philological research and analysis, we suggest reading the term Gospel as a transliteration from greek to hebrew. In the Babylonian Talmud, tractate Sabbath 116a-b, a verse from the Gospel of Matthew is brought with a number of distortions. If we note the patristic attestations on the hebrew original Gospel of Matthew, we can assume that a number of tannaim from the beginning of the second century, were familiar with fragments of Gospel of Matthew in the original hebrew. In this hypothesis is accepted, we can reveal a hidden $R$. Gamaliel polemic against a number of verses of the Gospel of Matthew and in general against jewish-christianity. 
Il est aujourd'hui attesté par une grande partie de la recherche que les Sages du Talmud, certainement à l'initiative de R. Gamaliel II de Yabneh, ont exclu les groupes judéo-chrétiens de la Synagogue. Cette démarche marquant la rupture entre juifs d'obédience pharisienne et juifs adeptes du mouvement de Jésus intervint à la fin du $\mathrm{I}^{\mathrm{er}}$ siècle. Elle est manifestée par une malédiction liturgique du nom de Birkat ha-minim couramment traduite par «bénédiction des hétérodoxes (ou des hérétiques)». Ce processus d'exclusion a marqué de son empreinte le regard des Sages du Talmud sur les juifs d'obédience messianique croyant en Jésus ${ }^{1}$.

La question de la Birkat ha-minim a été l'objet de nombreux travaux tendant à explorer sa formulation, son objet, ses destinataires ou encore sa percée historique ${ }^{2}$. Il convient toutefois de remarquer que la question de savoir dans quelle mesure les Sages connaissaient dès le $\mathrm{II}^{\mathrm{e}}$ siècle des fragments d'écrits chrétiens n'a quasiment pas mobilisé les critiques. Ce dossier pourtant crucial permet de comprendre sous de nouveaux éclairages les relations entre les Sages du Talmud, et particulièrement les tannaïm et les adeptes juifs du mouvement de Jésus. Ces adeptes, juifs de naissance, dont les conceptions religieuses deviendront progressivement après 70 un délit de pensée.

La présente étude a comme objectif d'essayer de donner des éléments de réponse à plusieurs questions : Dans quelle mesure les Sages du Talmud, à une époque aussi reculée que le $\mathrm{II}^{\mathrm{e}}$ siècle avaient-ils connaissance de textes se rapportant aux Évangiles? Quelles étaient, dans le monde chrétien, la nature et la constitution des Évangiles tétramorphes à cette même époque? Est-il possible de déceler des mentions scripturaires propres à la littérature néotestamentaire dans le corpus talmudique?

1. Nous renverrons ici à notre ouvrage: D. Jaffé, Le judaïsme et l'avènement du christianisme. Orthodoxie et hétérodoxie dans la littérature talmudique $I^{e r}$-II siècle, Paris, 2005.

2. Cf. notre étude de la Birkat ha-minim et la bibliographie citée en D. Jaffé, Le Talmud et les origines juives du christianisme. Jésus, Paul et les judéo-chrétiens dans la littérature talmudique, Paris, 2007, p. 121-135 et, en dernière analyse, voir l'ouvrage consacré à cette question de Y. Y. Tepler, Birkat haMinim, Tübingen, 2007. 
Dans un premier temps, on citera les témoignages textuels du corpus talmudique qui laissent entrevoir la mention du mot Évan-

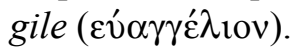

\section{État de la recherche}

Le premier passage qui doit être cité provient de la Tosefta Sabbath XIII, $5^{3}$ :

[En cas d'incendie], on ne sauve pas les guilyonim et les livres des Minim, ils brûlent sur place avec les mentions [du Nom de Dieu qu'ils renferment]. R. Yossi le Galiléen ${ }^{5}$ dit : «Les jours de semaine, on se met à lire ${ }^{6}$ les mentions [du Nom de Dieu], et on les met à l'abri, tandis qu'on brûle le reste ». R. Tarfon déclare : «Que je sois privé de mes enfants [plutôt que de manquer], si [ces livres] tombaient dans mes mains de les brûler, eux, et les mentions [du Nom de Dieu qu'ils renferment], car si l'on me poursuit, j'entrerai ${ }^{7}$ dans un lieu d'idolâtrie mais je n'entrerai pas ${ }^{8}$ dans leurs maisons, car les idolâtres (ser-

3. Selon le manuscrit d'Erfurt (Ed. M. S. Zuckermandel, p. 129). Voir pour les variantes de ce passage : Sifré Nasso (Éd. H. S. Horovitz, p. 21); Talmud de Jérusalem, Sabbath XVI, 1, 15c; Midrash Tanhuma (ajout à la péricope Korah 1; éd. S. Buber, p. 94-95); Talmud de Babylone, Sabbath 116a.

4. Ces mots sont absents du manuscrit d'Erfurt et du manuscrit de Londres (British Museum Add. 27. 296), en revanche ils se trouvent dans le manuscrit de Vienne. Voir S. Lieberman, The Tosefta, according to Codex Vienna with Variants from Codices Erfurt, London, Genizah Mss. and Edito Princeps (Venice 1521), Jérusalem, $1992^{2}$, p. 58.

5. Dans la première édition de la Tosefta (Compendium talmudique d'Alfasi, V) ainsi que dans le manuscrit de Vienne (S. Lieberman, The Tosefta, p. 5859), on trouve R. Yosa à la place de R. Yosi cependant cette substitution est certainement due à une erreur de copiste.

6. Le verbe koreh est certainement à corriger en koder qui signifie « découper » et correspond mieux au contexte. C'est celui-ci qui apparaît dans le manuscrit de Vienne (S. Lieberman, The Tosefta, p. 58). Selon S. Lieberman, Tosefta KiFshutah. A Comprehensive Commentary on the Tosefta, Jérusalem, $1992^{2}$, p. 207, koreh est une erreur propre au manuscrit d'Erfurt. Cf. aussi G. Alon, Histoire des juifs en Palestine durant l'époque de la Mishna et du Talmud, Tel-Aviv, 1975, vol. I, p. 181 note 306, [en hébreu], qui suppose également une erreur de copiste (Notons que cette note n'apparaît pas dans l'édition anglaise; cf. G. Alon, The Jews in their Land in the Talmudic Age, Jérusalem, 1980, vol. I, p. 291). Cette suggestion s'en trouve renforcée par les explications apportées par A. Kohut, Aruch Completum sive Lexicon Vocabula et Res, quae in Libris Targumicis, Talmudicis et Midraschicis, Vienne, 1878-1892, vol. I, p. 51-52; vol. VII, p. 63, qui cite ce passage et estime que la lecture exacte de koder est koded qui a le sens de « découper».

7. Dans le manuscrit de Londres (British Museum Add. 27. 296) on trouve la forme plurielle « on entre ».

8. Dans le manuscrit de Londres, c'est encore la forme plurielle négative « on n'entre pas » qui est mentionnée. 
viteurs de dieux étrangers) ne Le connaissent pas et Le renient alors qu'eux Le connaissent et Le renient ». Et c'est pour eux que le verset dit: «Derrière la porte et les linteaux, tu as installé ton mémorial » (Is 57, 8). R. Ismaël dit : «Puisque pour faire la paix entre un homme et sa femme, Dieu dit: Que mon Nom ${ }^{9}$ écrit dans la sainteté soit effacé avec de l'eau; les livres des Minim qui entraînent l'inimitié, la jalousie et les dissensions entre le peuple juif et son Père qui est aux cieux, à plus forte raison pourra-t-on les briser ${ }^{10}$, eux, et les mentions [du Nom de Dieu qui s'y trouvent] ». Et c'est pour eux que le verset dit : « Certainement, je hais ceux qui te haïssent, et ceux qui se dressent contre toi, je les déteste. Je les hais infiniment, je les considère comme des ennemis » (Ps 139, 21-22). Et de même qu'on ne les sauve pas d'un incendie, on ne les sauve pas non plus d'un éboulement, d'une inondation et de tout ce qui pourrait les perdre.

L'une des questions fondamentales liées à ce passage est l'identification du terme «guilyonim»(גיליונים). Ce terme a fait l'objet d'importantes études depuis le $\mathrm{XIX}^{\mathrm{e}}$ siècle. Parmi les critiques s'étant intéressés à son analyse, nombreux sont ceux qui proposent d'y voir la corrélation guilyonim/Évangile. C'est par exemple le cas avec J. H. Schorr ${ }^{11}$, J. M. Jost ${ }^{12}$, J. Derenbourg ${ }^{13}$, A. H. Goldfahn $^{14}$, H. Graetz ${ }^{15}$, L. Goldschmidt ${ }^{16}$ et B. W. Helfgott ${ }^{17}$. Selon cette approche, le vocable « guilyonim » serait une translittération

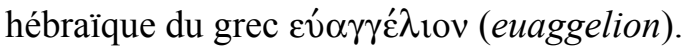

Selon L. Blau, le terme "guilyonim » fait référence aux Évangiles sans être porteur d'une quelconque connotation péjo-

9. Dans le manuscrit de Vienne (S. Lieberman, The Tosefta, p. 58), on trouve la formule : « Que le livre ».

10. Dans le manuscrit de Vienne (S. Lieberman, The Tosefta, p. 59), on trouve «pourra-t-on les effacer». S. Lieberman, Tosefta Ki-Fshutah, p. 207, a montré que la version exacte du manuscrit d'Erfurt est «brûler » qui signifie « pourra-t-on les brûler » que l'on retrouve dans le manuscrit de Londres.

11. Cf. J. H. Schorr, «Shegagot Talmud », dans Hehalutz 1 (1852), p. 57.

12. Cf. J. M. Jost, Geschichte des Judentums und Seinersekten, Leipzig, 1858, vol. II, p. 39-40.

13. Cf. J. Derenbourg, Essai sur l'histoire et la géographie de la Palestine, d'après les Talmuds et les autres sources rabbiniques, Paris, 1867, p. 379-380.

14. Cf. A. H. Goldfahn, « Über den Ursprung und die Bedeutung des Ausdruckes «Min» im babyl. und jerus. Talmud», dans Monatsschrift für Geschichte und Wissenschaft des Judentums 19 (1870), p. 169.

15. Cf. H. Graetz, History of the Jews from the Reign of Hyrcanus (135 B. C.E) to the Completion of the Babylonian Talmud (500 C.E), Philadelphie, 1893, p. 378-379.

16. Cf. L. Goldschmidt, Der Babylonische Talmud mit Einschluss der Vollstaendigen Misnah, Berlin, 1925, p. 598.

17. Cf. B. W. Helfgott, The Doctrine of Election in Tannaitic Literature, New York, 1954, p. 85. 
rative. De plus, ce critique suppute que la forme brève הגליון (haguil(a)yon) devait être la plus ancienne et définissait non pas plusieurs Évangiles différents, mais plutôt diverses copies du même Évangile ${ }^{18}$.

C. C. Torrey admet également la relation guilyonim/Évangiles en considérant que seule une étude philologique et sémantique peut permettre de clarifier l'origine de ce cryptogramme. Dans cet esprit, ce critique se demande pourquoi précisément un terme grec tel euaggelion désignerait les Évangiles dans la littérature talmudique. En effet, il est difficile de penser que les Sages du Talmud rédacteurs de ce passage de la Tosefta Sabbath aient délibérément choisi un vocable issu d'une langue étrangère afin de qualifier les Évangiles. Il est tout aussi difficilement imaginable que les juifs disciples de Jésus considérant les Évangiles en tant que livres inspirés se soient servis d'un terme grec pour qualifier ces textes. Ces deux problématiques énoncées, Torrey développe l'idée suivante : בשורא (besora) pour rendre euaggelion. En outre, ce même terme araméen est rendu par ce même terme grec dans certains passages de la Sep-

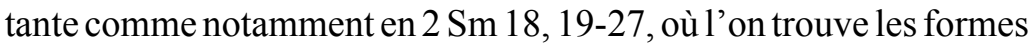

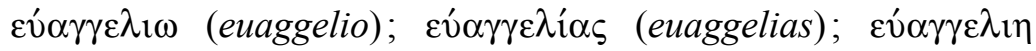

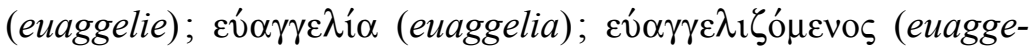

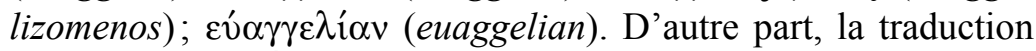
araméenne de l'Évangile selon Marc est introduite par les mots : (rech besoratha di yeshua meshih'a) qui signifient littéralement "Commencement de la nouvelle de Jésus le Messie » et qui est rendue en grec par 'Ap

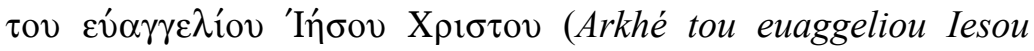
Khristou). Au niveau du contexte historique, les judéo-chrétiens utilisaient certainement le terme araméen besorah pour désigner les Évangiles. Or, selon Torrey, les Sages se sont radicalement opposés à l'emploi d'un terme hébraïco-araméen pour qualifier un corpus littéraire qui sera progressivement défini comme dissident de leurs normes. Par voie de conséquence, quand les judéo-chrétiens seront considérés comme officiellement déviants, assimilés aux païens et définitivement exclus, les Sages préféreront opter pour le terme grec euaggelion en le translittérant en hébreu, plutôt que pour le

18. Cf. L. Blau, art. "Gilyonim», dans The Jewish Encyclopedia, New York/Londres, $1925^{3}$, vol. V, p. 668. 
terme besorah jugé comme chargé d'un fondement biblique trop profond $^{19}$.

G.F. Moore met en évidence le sens originel du terme 《guilyonim»(גיליונים) qui se rapporte aux parties non écrites ou encore aux espaces blancs d'un parchemin. De la sorte, il montre que la traduction la plus plausible de ce cryptogramme serait certainement « marge ». De cette manière, il suppose que la littérature talmudique s'est livrée à une extension entre la signification première de ce vocable et le message que l'on voulait transmettre des Évangiles. Ainsi, la démarche aurait été de mettre sur un même pied d'égalité les blancs des rouleaux de parchemin et le caractère vide des Évangiles. Il s'agirait ainsi d'un véritable glissement sémantique qui aurait été réalisé par les Sages afin d'insister sur l'aspect futile de l'Évangile. En outre, selon G. F. Moore, les amoraïm de la Baraïta de Sabbath 116a étaient ignorants de l'origine du terme "guilyonim ». En revanche, ils connaissaient son sens initial de « marges » ou « espaces blancs » et l'auraient affilié aux Évangiles pour faire ressortir le fait que les «Évangiles ne sont rien de plus qu'un espace blanc $»^{20}$.

En effet, on peut se demander à juste titre dans quelle mesure les amoraïm de la Baraita de Sabbath 116a et du TJ Sabbath XVI, $1,15 \mathrm{c}$, connaissaient la signification initiale de « guilyonim ». Tout porte à croire que tel ne fut pas le cas, notamment par la question de savoir si l'on parle des guilyonim d'un rouleau de la Torah rapporté par la Baraïta. De fait, il est possible de penser que ce terme a été retranscrit dans les versions judéennes et babyloniennes des Talmud alors que les amoraïm n'en possédaient plus le sens ${ }^{21}$. Si l'on

19. Cf. C. C. Torrey, Documents of the Primitive Church, New York/ Londres, 1941, p. 100-101. Notons que ce critique n'exclut cependant pas que « guilyionim » dans sa forme singulier « guilayion » puisse, dans certains contextes désigner également les marges blanches du parchemin d'un rouleau (Voir Id, Ibid, p. 101 note 55$)$.

20. Cf. G. F. Moore, « The Definition of the Jewish Canon and the Repudiation of Christian Scriptures ", dans Essays in Modern Theology and Related Subjects, New York, 1911, p. 115-141 (= S. Z. Leiman (Ed.), The Canon and Masorah of the Hebrew Bible. An Introductory Reader, New York, 1974, p. 115-141), spécialement p. 105.

21. Cf. L. Ginzberg, « Some Observations on the Attitude of the Synagogue towards the Apocalyptic-Eschatological Writings », dans Journal of Biblical Literature 41 (1922), p. 115-136, et spécialement p. 122-123 note 19 (= J. B. Agus (Ed.), Judaism and Christianity Selected Accounts 1892-1962, New York, 1973, p. 122-123 note 19; S. Z. Leiman (Ed.), The Canon and Masorah of the Hebrew Bible, p. 142-163 et spécialement p. 149-150 note 19). 
accepte cette lecture, on doit donc conclure que la juxtaposition de גיליונים (guilyonim) avec סיפרי מינים (sifre minim) ne signifie pas que chacun de ces deux termes possède son propre sens, mais plutôt qu'ils furent rapportés par les deux versions du traité Sabbath en se fondant sur la version de la Tosefta.

S. Lieberman estime au contraire que le sens de «guilyonim» était connu des amoraïm de la Baraïta de Sabbath 116a. Il fonde son propos en proposant une lecture différente du passage talmudique qui serait non pas גיליונים וסיפרי מינים (guilyonim vesifre minim) mais (guilyonim shel sifre minim) גיליונים של סיפרי מינסים c'est-à-dire « les guilyonim des livres des Minim » à la place des « guilyonim et les livres des Minim ». Cette lecture l'amène à penser que «guilyonim » se réfère aux Évangiles joints à la Torah. Ainsi, les Sages, au fait de la signification de ce cryptogramme, auraient statué sur les guilyonim/Évangiles en refusant de les sauver d'un incendie un jour de Sabbath, et ce, bien que se composant de mentions du Nom de Dieu. Finalement, la démarche halakhique concernant les « livres des Minim » est identique à celle relative aux guilyonim/Évangiles joints à la Torah. Dans tous les cas de figure, on ne sauve ni les uns ni les autres ${ }^{22}$.

Dans un autre de ces travaux, Moore ajoute quelques intéressantes remarques sur cette question. Il fait d'abord remarquer que la littérature talmudique ne témoigne d'aucun nom hébraïque définissant les Évangiles. À l'opposé de Torrey, ce critique affirme que le vocable « guilyonim » n'est pas le produit d'une traduction d'une locution sémitique mais la perpétuation d'un nom originel non hébraïque transmis par les Sages ${ }^{23}$.

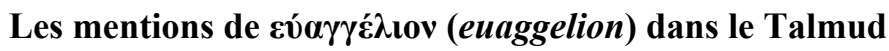

La mention de " guilyonim » se retrouve également dans un passage de TB Sabbath 116a. Ce texte qui est un parallèle de Tosefta Sabbath XIII, 5 fait apparaître aux noms de R. Meïr et de R. Yohanan d'autres propos relatifs aux « guilyonim ». Ainsi, on peut lire :

22. Cf. S. Lieberman, Tosefta Ki-Fshutah, p. 206 note 16.

23. Cf. G. F. Moore, Judaism in the First Centuries of the Christian Era. The Age of the Tannaim, Londres, 1962, vol. III, (6 édition), p. 67 note II. 
R. Meïr les appelait : «aven-guilyon », R. Yohanan les appelait : « avon-guilyon ».

Notons tout d'abord, que cette phrase n'apparaît pas dans les éditions courantes du Talmud de Babylone du fait de la censure chrétienne s'étant exercée au Moyen Âge ${ }^{24}$. On peut déjà souligner que ce propos se compose d'un commentaire cacophémique sur le terme « guilyonim ». Il semble s'agir en fait d'une distorsion du

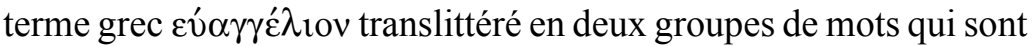
אוון גליון (aven guilayon) et עוון גליון (avon guilayon). Dans la glose de Rachi sur ce passage, il est stipulé qu'il est fait référence aux livres des Minim que R. Meïr nommait aven guilayon du fait qu'ils [les chrétiens] 1'appelaient אוונגיל"א (evangila ${ }^{25}$. Il semblerait donc que Rachi ait voulu expliquer le propos de R. Meïr en le retranscrivant afin de former une glose en français. À cet égard, il paraît ne faire aucun doute que « evangila » soit relatif aux Évangiles.

Il sera donc question d'essayer de comprendre si les propos de R. Meïr et de R. Yohanan peuvent être expliqués en ce sens. Pour ce faire, il semble opportun de commencer à s'interroger sur la signification des préfixes aven et avon au travers d'une étude lexicographique.

Dans la littérature biblique, le substantif aven peut exprimer le mal, l'iniquité, le mensonge, la tromperie, l'injure, la calamité, le châtiment, l'injustice, la déception et le culte idolâtre. Le terme

24. Il faut donc avoir recours aux manuscrits ainsi qu'au corpus de R. Rabbinowicz, Diqduqé Soferim, Variae Lectiones in Mishnam et in Talmud Babylonicum, Munich, 1868-1886, traité Sabbath, p. 260. Dans la variante de ce passage, R. Rabbinowicz note de Rabbi Meir ce qui voudrait dire qu'il s'agit non de R. Meïr lui-même mais de l'école de R. Meïr. Ceci reviendrait à dater ce dictum à une date plus tardive.

25. Notons que cette glose n'apparaît pas non plus dans les éditions courantes. Voir R. Rabbinowicz, Diqduqé Soferim, p. 260 note 60. Le mot « Evangila » apparaît sous différentes formes dans les manuscrits suivants : dans le Ms. 324 fond hébreu (ancien fond Sorbonne 220) on trouve aven guilayon avec une rature; dans le Ms. 2087 (Catalogue De-Rossi 1324) de la bibliothèque de Parme, la mention est avenilosh; dans le Ms. Vat. ebraico 138 (ancien Palatin) du Vatican, on lit ivenguilosh; dans le Ms. Oriental 5975 du British Museum de Londres, le Ms. Elkan N. Adler 1621 de la bibliothèque du Jewish Theological Seminary de New York, le Ms. du Jewish Theological Seminary de New York, les éditions du Talmud de Vilna et les éditions du Talmud de Soncino on trouve avenguila. Voir A. Darmesteter; D. S. Blondheim, Les gloses françaises dans les commentaires talmudiques de Raschi, Paris, 1929, vol. I, p. 62 nº 454. 
avon désigne l'iniquité, mais également la transgression, la culpabilité causée par la transgression, le châtiment comme conséquence de la faute, et le crime ${ }^{26}$. En ce qui concerne la littérature talmudique, le terme aven désignera quelque chose de creux, de vide mais aura également le sens de mensonge et de vanité. Concernant avon, il s'agira de l'idée d'erreur voire de perversion ${ }^{27}$.

Selon W. Bacher, le sens de aven est « injustice» ou « idolâtrie » alors que avon signifierait « péché ». Ce critique considère que R. Meïr et R. Yohanan s'emploient à la méthode du notaricon,

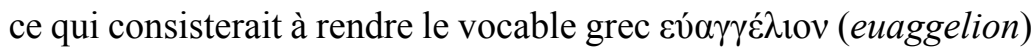
par deux termes hébraïques. Il ne fait aucun doute selon W. Bacher que les propos de ces deux Sages se rapportent aux guilyonim dont il est question dans la Baraita. Ainsi, guil(a)yon signifierait «Évangile» tandis que guilyonim «serait les exemplaires de ce livre $»^{28}$.

Selon R. T. Herford, R. Yohanan aurait eu à l'esprit le propos de R. Meïr et s'en serait inspiré afin d'en donner sa propre lecture. Pour ce critique, il est indéniable que le « jeu d'esprit » initial de R. Meïr autant que celui qui lui succède émanant de R. Yohanan désignent l'Évangile. Ce qui l'amène à conclure que le terme euaggelion était connu aux époques de ces deux Sages et qu'il avait même un usage commun. Il aurait pu ainsi avoir un sens générique afin de désigner les « livres des Minim » et faire référence à plusieurs corpus différents. ${ }^{29}$

26. Cf. E. ben Iehuda, Thesaurus Totius Hebraitatis et Veteris et Recentioris, Jérusalem, 1950, vol. I, p. 105-106; vol. IX, p. 4377-4380; L. Koehler; W. Baumgartner, The Hebrew and Aramaic Lexicon of the Old Testament, New York/Leyde, 1994, vol. I, p. 22 ; vol. II, p. 800 ; E. Carpenter; M. A. Grisanti, art. « awen », dans New International Dictionary of Old Testament Theology and Exegesis, Grand Rapids, 1997, vol. I, p. 309-315; A. Luc, art. « awon », dans Ibid, vol. III, p. 351; The Encyclopedic Dictionary of the Bible, Tel-Aviv, 1998, vol. I, p. 37; vol. IV, p. 868, [en hébreu]; The Brown-Driver-Briggs Hebrew on English Lexicon, Boston, 1999 (4e édition), p. 19-20; 730-731. Notons que dans le livre d'Osée, la forme composée beth aven se réfère à Beth-El et désigne la maison d'iniquité (Os 4,$15 ; 5,8 ; 10,5)$.

27. Cf. A. Kohut, Aruch Completum, vol. I, p. 45, qui présente aven uniquement comme le préfixe de la forme composée aven guilyon et M. Jastrow, $A$ Dictionary of the Targumim, the Talmud Babli and Yerushalmi, and the Midrashic Literature, Londres, 1903, p. 27; 1053.

28. Cf. W. Bacher, «Le mot «minim» dans le Talmud désigne-t-il quelquefois des chrétiens? », dans Revue des études juives 38 (1899), p. 39-40.

29. Cf. R. T. Herford, Christianity in Talmud and Midrash, Londres, 1903, p. $163-164$. 
D. Sperber propose une thèse similaire en expliquant que les jeux de mots satiriques opérés par R. Meïr et R. Yohanan sont la preuve que le terme «guilyonim » se réfère à l'Évangile. D'autre part, il propose de traduire aven guil(a)yon par « rouleau de mensonge » et avon guil(a)yon par « rouleau de transgression ». Par voie de conséquence, en dépit des mentions du Nom de Dieu et des versets scripturaires qu'ils contiennent, ces rouleaux ne peuvent être sauvés d'un incendie un jour de Sabbath ${ }^{30}$.

De fait, bien qu'à la lecture du passage de la Tosefta, il subsistât certaines incertitudes quant à l'identification des " guilyonim » en tant qu'Évangiles, force est de reconnaître qu'avec les propos de R. Meïr et de R. Yohanan, cette affiliation ne semble plus faire aucun doute. Cela ne veut pas dire pour autant que les « guilyonim » expriment systématiquement une relation avec les Évangiles. En fait, on peut postuler pour un glissement sémantique entre les deux sens de ce terme. Une forme de transition qui se serait effectuée entre le sens initial de guilyonim/marges ou espaces blancs, puis le sens second de guilyonim/Évangiles. Il devient donc possible de proposer le schéma suivant : dans les passages susmentionnés, les «guilyonim » sont relatifs aux Évangiles, alors qu'à l'origine le sens de ce vocable n'était autre que celui de "marges» ou «d'espaces blancs ». La question est donc maintenant de savoir pourquoi justement un glissement s'est opéré entre ces deux sens? On doit d'emblée commencer par dire que les distorsions du terme grec euaggelion effectuées par R. Meïr et par R. Yohanan viennent éclairer la signification du terme "guilyonim » jusque-là cryptée. R. Meïr se fonde sur le sens initial de marges ou d'espaces blancs et estime que les Évangiles ne sont autres qu'un rouleau vide de sens, voire un rouleau de mensonge; alors que pour R. Yohanan les Évangiles sont à définir comme un rouleau de transgression. Il semblerait qu'avec ce dernier une étape soit franchie, car on passe de l'idée de vacuité à celle de transgression religieuse avec toutes les conséquences que cela implique ${ }^{31}$. Dans cette perspective, il devient possible de subodorer que les rédacteurs du passage de la

30. Cf. D. Sperber, art. "Sifrei ha-minim », dans Encyclopaedia Judaica, Jérusalem, 1971, vol. XIV, p. 1521.

31. Voir les intéressantes remarques d'ordre littéraire allant dans le sens de cette analyse chez R. Gibbs; D. Jacobson; J. Diamond, « Empty Margins? », dans Conservative Judaism 42 (1989/1990), p. 21-33 et spécialement p. 28. 
Tosefta devaient connaître les propos de R. Meïr en faisant usage du terme "guilyonim». En conséquence, ce terme se composait déjà en filigrane de l'approche de R. Meïr. En résumé, il devient envisageable de faire la lecture suivante du passage de T Sabbath XIII, 5 : « [En cas d'incendie], on ne sauve pas les Évangiles car ils n'ont aucune valeur et sont même emplis de mensonges ».

À ce stade de la recherche, au moins deux remarques peuvent être émises :

1. La combinaison des termes aven et avon avec guil(a)yon est une

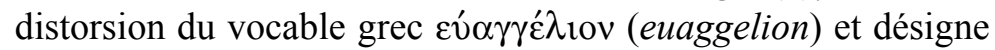
גליון (guil(a)yon) n'ait pas ce sens mais plutôt celui de « marges » ou «d'espaces blancs » ${ }^{32}$.

2. À la fin de la première moitié du II ${ }^{\mathrm{e}}$ siècle, R. Meïr devait avoir connaissance de l'existence d'une forme certainement partielle des Évangiles. Il savait les rattacher aux judéo-chrétiens et se positionner à leur égard.

Ces remarques formulées, il convient cependant de s'interroger encore sur un point. En effet, on ne pourra leur donner toute leur

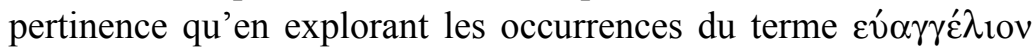
(euaggelion) dans les sources chrétiennes. La question qui retiendra notre attention sera donc de savoir si au $\mathrm{II}^{\mathrm{e}}$ siècle, le terme euaggelion pouvait désigner les Évangiles.

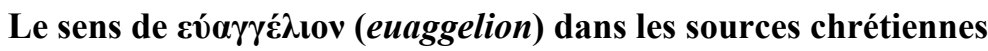

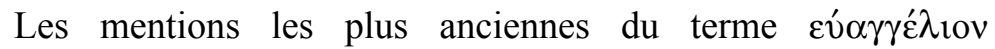
(euaggelion) figurent dans le corpus paulinien. Dans cette littérature, son emploi semble être celui d'un terme technique servant à édifier le message christique et surtout à élaborer sa proclamation. Il est à présumer que ses racines sont à chercher dans l'activité missionnaire de Paul de Tarse ${ }^{33}$. Notons que ce terme n'apparait ni

32. Cette assertion peut être affermie par la suite du passage de Sabbath 116a-b où il est question d'un philosophe judéo-chrétien corrompu, en contact avec R. Gamaliel et sa sœur Imma Chalom afin de statuer sur un cas d'héritage. Ce passage dans lequel le philosophe s'appuie sur le avon guilyon est analysé par la suite.

33. Sur la question de ce terme dans les Épîtres pauliniennes, voir W. H. Kelber, Tradition orale et écriture, Paris, 1991, p. 201-219. 
dans l'Évangile de Luc ni dans celui de Jean, cependant sa mention dans la littérature gréco-romaine dans le sens de "nouvelle» ou de «bonne nouvelle » semble avoir influencé l’Évangile selon Matthieu, l'Évangile selon Marc ainsi que les Actes des Apôtres. En outre, dans ces textes la signification de ce terme s'étendra au travers du triptyque : proclamation, prédication, audition ${ }^{34}$. On peut également souligner que le terme euaggelion se trouve dans les formules «Évangile de Dieu », «Évangile du Christ », «Évangile de Notre Seigneur » ou encore «Évangile du Royaume $»^{35}$.

Il convient de noter que l'emploi de ce terme est également fréquent dans la littérature apostolique. Cependant, il est difficile de déclarer s'il désigne les Évangiles dans leur forme définitive. Il semblerait plutôt que leur usage se réfère au sens paulinien, à savoir celui d'immédiateté et de dynamisme dans la proclamation orale du message christique. Dans cette optique, R. H. Gundry estime que la littérature apostolique utilise des livres qui sont devenus

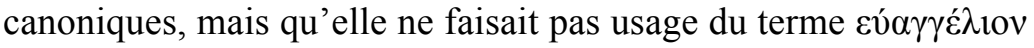
(euaggelion) afin de les qualifier ${ }^{36}$. Cela reviendrait à dire que dans la littérature apostolique du $\mathrm{II}^{\mathrm{e}}$ siècle, ce terme n'avait alors pas l'usage commun d'Évangile. H. Koester renforce cette approche en supposant que certains auteurs ont assemblé leurs matériels évangéliques de documents écrits, toutefois cela ne signifie pas pour autant qu'ils aient appelé ces documents euaggelion dans le sens d'Évangile ${ }^{37}$.

Il semblerait que la première occurrence de euaggelion afin de définir un texte écrit se trouve dans l'œuvre de Justin de Naplouse. En effet, bien que Justin se réfère usuellement à la vie et à l'enseignement de Jésus sous la formule : « la mémoire des apôtres » ou sim-

34. Cf. Pour proclamation : Mt 4, 23; 9, 35; 24, 14; 26, 23 ; Mc 1, 14; 13 , $10 ; 14,9 ; 16,5 ;$ Ga 2, 2; Col 1, 23; 1 Th 2, 9. Pour prédication : 1 Co 9, 8; 15, 1 ; 2 Co 11, 7; Ga 1, 11; Ap 14, 6. Pour audition : Ac 15, 7; Col 1, 23 ; Ep 1, 13.

35. Cf. Pour «Évangile de Dieu»: Mc 1, 14; Rm 1, 1; 15, 16; 1 Th 2, 2; 8, 9; $1 \mathrm{P} \mathrm{4,} \mathrm{17;} 1 \mathrm{Tm} 1$, 11. Pour «Évangile du Christ »: Rm 15, 19; 1 Co 9, 12; 2 Co 2,$12 ; 9,13 ; 10,14 ;$ Ga 1,$7 ; \mathrm{Phm} 1,27 ; 1$ Th 3, 2; et aussi sous une forme différente en : Mc 1, 1; Rm 1, 9; 2 Th 1, 8; 2 Co 4, 4. Pour « Évangile de Notre Seigneur » : Did 15, 4 (trad. W. Rordorf; A. Tuilier, Paris, 19982, Sources Chrétiennes $n^{\circ} 248$ bis, p. 195). Pour « Évangile du Royaume » : Mt 4, 23; 9, 35; 24, 14.

36. Cf. R. H. Gundry, «ЕҮАГГЕ $\Lambda$ ION : How Soon a Book? », dans Journal of Biblical Literature 115 (1996), p. 321-325.

37. Cf. H. Koester, Ancient Christian Gospels : Their History and Development, Londres, 1990, p. 15; 17-18; 22-23. 
plement « la mémoire », il fait usage de ce terme à trois reprises ${ }^{38}$. Il est toutefois malaisé de définir précisément à quelle forme de document Justin se réfère ${ }^{39}$. Certains critiques ont supposé que Justin a délibérément évité d'employer le terme euaggelion, bien qu'il ait été de plus en plus usité durant le $\mathrm{II}^{\mathrm{e}}$ siècle. Cette réticence s'explique peut-être par le fait que ce terme semble désigner à cette époque des textes individuels plutôt qu'un corpus complet, ${ }^{40}$ et plus certainement par la lutte s'élaborant contre le marcionisme en expansion $^{41}$. À ce propos, on doit souligner que Marcion semble avoir été le premier à utiliser le terme euaggelion en référence à un livre ${ }^{42}$, peut-être d'ailleurs à cause d'une interprétation erronée de la formule paulinienne « mon Évangile » $(\mathrm{Rm} 2,16)$ perçue comme un document spécifique ${ }^{43}$. Ainsi, à l'appui des corrections effectuées dans l'Évangile selon Luc qui consistèrent principalement à retirer les éléments imprégnés de judaïsme, Marcion et ses disciples clamèrent la possession d'un Évangile « authentique $»^{44}$.

38. Cf. Justin de Naplouse, Dialogue avec le juif Tryphon, 10, 2; 100, 1 (trad. G. Archambault; L. Pautigny, Paris, 1994, p. 113; 255; P. Bobichon, Justin Martyr, Dialogue avec Tryphon, Fribourg, 2003, vol. I, p. 209). Le premier passage met ce terme dans la bouche de Tryphon qui traite les commandements de « ce

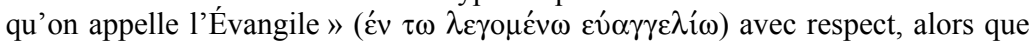
dans le second, ce terme est utilisé afin d'introduire un propos de Jésus. Notons que ce procédé se trouve également en 2 Clem 8, 5 et 1 Apol 15-17.

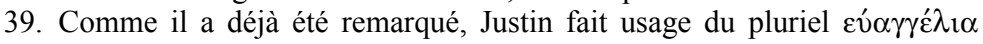
(euaggelia) en 1 Apol 66, 3. Cependant, dans ce cas il peut s'agir d'un document individuel. Voir à ce propos O. Piper, "The Nature of the Gospel according to Justin Martyr », dans Journal of Religion 41 (1961), p. 155; 162-163. Selon P. Bobichon, Justin Martyr, Dialogue avec Tryphon, vol. II, p. 607 note 6, ce terme désigne non pas les « sources de l'histoire du Christ » mais plutôt l'Évangile du Seigneur, évoqué dans la Didaché $(8,2 ; 15,4)$ qui contenait essentiellement des instructions pratiques dont le but était de fixer la conduite des individus et des communautés en diverses circonstances.

40. Cf. C. H. Cosgrove, «Justin Martyr and the Emerging Christian Canon : Observations on the Purpose and Destination of the Dialogue with Trypho », dans Vigiliae Christianae 36 (1982), p. 221-223.

41. Cf. H. Koester, Ancient Christian Gospels, p. 37-39; C. H. Cosgrove, " Justin Martyr and the Emerging Christian Canon », p. 225.

42. Cf. H. Koester, Synoptische Überlieferungen bei den Apostolischen Vätern, Berlin, 1957, p. 6-12; H. Koester, « From the Kerygma-Gospel to Written Gospels ", dans New Testament Studies 35 (1989), p. 361-381; H. Koester, Ancient Christian Gospels, p. 1-43.

43. Cf. H. Koester, Ancient Christian Gospels, p. 35-37; O. Cullmann, The Early Church, Londres, 1956, p. 48; H. Von Campenhausen, The Formation of the Christian Bible, Philadelphie, 1972, p. 153-156.

44. Cf. B. Metzger, The Canon of the New Testament, Oxford, 1987, p. 92. 
À cette même époque, Irénée de Lyon emploie lui aussi le terme euaggelion. Il est vrai qu'il n'est cependant pas toujours aisé de définir l'aspect que peut avoir ce terme dans le Contre les hérésies. En effet, on peut le trouver faisant indifféremment référence à un corpus oral ou écrit. Cependant, il est important de souligner qu'Irénée est le premier à définir les quatre Évangiles sous la forme evangelium ${ }^{45}$. Certains ont d'ailleurs vu dans cette mention la preuve d'une transition depuis une tradition orale vers une tradition écrite qui justement marquerait le passage progressif vers la rédaction de l'Évangile ${ }^{46}$. D'autres ont quelque peu radicalisé cette approche en estimant que la mention des quatre évangiles d'Irénée censée décrire l'Évangile suppose dès cette époque, l'acceptation de ces textes en tant que canon chrétien. Ainsi, dès les années 170-180, il serait déjà question de l'existence d'un codex standardisé étant le support du canon contenant les quatre Évangiles ${ }^{47}$. Si tel est le cas, nous serions en présence du premier témoignage chrétien se référant au canon des quatre Évangiles en faisant usage du terme euaggelion ${ }^{48}$.

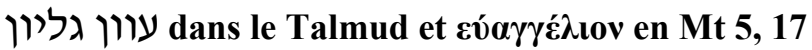

Il convient à présent de mentionner un passage très important qui mentionne les termes avon guil(a)yon en référence claire au corpus évangélique. Ce passage représente un témoignage d'une nature très particulière et possède une seule occurrence mentionnée en Sabbath 116a-b. Ce passage est certainement de rédaction tar-

45. Cf. Contre les hérésies III, 11, 8 (trad. A. Rousseau, p. 314-315).

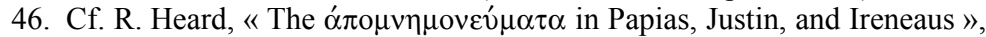
dans New Testament Studies 1 (1954), p. 129; H. Von Campenhausen, The Formation of the Christian Bible, p. 182, déclare qu'Irénée marque le passage de « la transition depuis une ancienne période de croyance en la tradition vers un nouvel âge de standardisation délibérée du canon ». Dans cette démarche voir aussi L. M. McDonald, The Formation of the Christian Biblical Canon, Peabody, $1996^{2}$, p. $164-165$.

47. Cf. T. C. Skeat, «Irenaeus and the Four-Gospel Canon », dans Novum Testamentum 34 (1992), p. 198-199; G. Stanton, « The Fourfold Gospel », dans New Testament Studies 43 (1997), p. 340, qui se montre plus prudent dans ses analyses.

48. Cf. A. Yoshiko Reed, "ЕҮАГГЕ $\Lambda \mathrm{ION}$ : Orality, Textuality, and the Christian Truth in Irenaeus' Adversus Haereses », dans Vigiliae Christianae 56

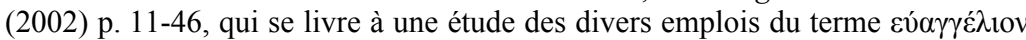
dans le Contre les hérésies et considère que ce terme ne qualifie pas obligatoirement le canon des quatre Évangiles mais plutôt un complément à la tradition orale déjà existante. 
dive puisque les termes avon guil(a)yon définissent sans équivoque les Évangiles et qu'il est écrit en araméen d'époque talmudique et non en hébreu d'époque tannaïtique. Ainsi, on peut lire :

Imma Shalom était la femme de R. Eliézer et la sœur de R. Gamaliel. Il y avait dans leur quartier un philosophe qui avait la réputation de ne jamais se laisser corrompre. Ils voulurent se moquer de lui. [Imma Shalom] lui apporta une lampe en or. Ils [R. Gamaliel et Imma Shalom] vinrent chez lui. Elle lui dit : « Je veux que l'on me donne une part des biens familiaux ». Il leur dit : «Partagez». R. Gamaliel lui dit : «Il est écrit 'Quand il y a un fils, la fille n'hérite pas ». Il lui dit : « Depuis le jour où vous avez été exilés de votre terre, la loi de Moïse a été prise $^{49}$ et la loi de l'Évangile a été donnée ${ }^{50}$ (l'a remplacée), or, il est écrit [dans cette loi] : "le fils et la fille hériteront à parts égales" ». Le lendemain, R. Gamaliel lui apporta un âne de Lybie. Il leur dit [le philosophe] : « J'ai consulté la fin de l'Évangile" ${ }^{51}$ et il y est écrit : "Je ne suis venu ni pour retrancher à la loi de Moïse, ni pour ajouter à la loi de Moïse ${ }^{52}$ ", et il est écrit [dans cette loi] : "Quand il y a un fils, la fille n'hérite pas" ». [Imma Shalom lui dit] : «Que ta lumière brille comme une lampe ». R. Gamaliel lui dit : «L'âne est venu et a donné un coup de pied à la lampe ».

Une fois les principaux personnages de l'histoire présentés, le passage énonce clairement l'objectif de la démarche : Imma Shalom et $\mathrm{R}$. Gamaliel veulent tourner en dérision un juge. La moquerie dont il fait l'objet n'est pas vaine, elle vise à montrer son caractère corruptible et à remettre en question sa réputation. Il ne s'agit donc pas d'obtenir de lui une sentence sur un problème juridique, mais plutôt d'orienter la démarche vers une polémique dont les enjeux semblent de grande importance.

On a l'impression, à la lecture du début du passage, que ce n'est pas tant la personne du juge en sa qualité de décideur qui intéresse

49. Dans le manuscrit d'Oxford, on trouve la mention : « la loi de Moïse a été prise de vous et la loi de l'Évangile vous a été donnée ».

50. Dans certains manuscrits, on trouve la mention: « et une autre loi a été donnée ». Voir R. Rabbinowicz, Diqduqé Soferim, traité Sabbath, p. 261. Notons que dans les éditions imprimées, il figure : "et un autre livre a été donné». La mention avon guil(a)yon (Évangile) apparaît dans les anciennes éditions du Talmud ainsi que dans le manuscrit de Munich. Il semblerait que les autres versions correspondent aux textes censurés.

51. Ici encore, dans les éditions imprimées, on trouve : " la fin du livre ».

52. Dans les anciennes éditions du Talmud, on trouve (mais) à la place de ולא (ni); cette correction est capitale car elle change la lecture de la citation. Ainsi, on doit lire : «Je ne suis pas venu pour retrancher à la loi de Moïse, mais pour ajouter à la loi de Moïse ». Notons que cette correction est attestée comme étant la version primitive. Voir R. Rabbinowicz, Diqduqé Soferim, p. 261 note aleph. 
Imma Shalom et R. Gamaliel, mais plutôt sa représentativité (dont on ne sait d'ailleurs pas encore ce qu'elle détermine). Il sera donc question de ridiculiser, non pas seulement un personnage, mais également le groupe d'affiliation auquel il se rattache.

Ainsi, avant toute forme de dialogue, avant même que l'objet du problème soit émis, ils lui apportent une lampe en or, objet de corruption. Cet objet signifie par sa nature et sa position dans le texte, le manque de rectitude du juge, trait de caractère que le récit va s'évertuer à mettre en évidence de façon continue. Il en sera même la trame principale. Une fois la question posée et la réponse du juge obtenue, survient la contradiction de R. Gamaliel qui se fonde sur la priorité du fils sur la fille dans l'héritage familial. Cet argument s'oppose à la décision du juge qui permet l'héritage de la fille au même titre que celui du fils. Notons que la citation de R. Gamaliel ne figure pas dans la Bible sous cette forme, mais semble être une déduction sous forme d'extrapolation de $\mathrm{Nb} 27,8:$ «(..) Si un homme meurt sans laisser de fils, vous ferez passer son héritage à sa fille ». Il faut remarquer que Imma Shalom et R. Gamaliel étant frère et sœur, le cas de figure posé peut avoir une valeur réelle du point de vue de la halakha. Cependant, tout concorde pour considérer que seule la forme présente une réalité casuistique concrète, la question ne servant que de support casuel abstrait; l'objet de la démarche reste la dérision.

À ce moment du récit, intervient un autre argument du juge qui, dans un premier temps, ne se fonde pas sur une citation ou une interprétation scripturaire. Il s'agit d'autre chose : montrer la caducité de la Loi sur laquelle s'appuie R. Gamaliel et par voie de conséquence, de nier son argumentation. La phrase : «Depuis le jour où vous avez été exilés de votre terre, la loi de Moïse a été prise et la loi de l'Évangile a été donnée », est à entendre comme l'état d'une transition propre à la révélation divine et à ses répercussions sur le peuple juif anciennement dépositaire de la Loi. L'exil de la terre d'Israël a donc entraîné la désuétude de la Loi mosaïque à laquelle fut substitué l'Évangile. C'est donc sur cette nouvelle lo $i$ que le juge se fonde pour statuer sur l'héritage «à parts égales » de la fille.

Il est très intéressant de noter que le texte mentionne des points de doctrine capitaux relatifs aux premières polémiques entre juifs et chrétiens. On peut à ce titre citer au moins trois notions : 1/ L'exil 
de la terre d'Israël comme châtiment divin. 2/ La suppression de la Loi de Moïse et sa caducité. 3/ La substitution de cette loi par l'Évangile : nouvelle alliance d'une nouvelle loi révélée.

Deuxième partie du récit: ce n'est plus Imma Shalom mais R. Gamaliel qui apporte au juge un objet pour le corrompre. Il s'agit d'un âne de Lybie qui, comme on pouvait le supposer, va l'influencer dans son jugement. Ici encore, c'est en fonction de la lecture de la loi (la nouvelle loi) que sera établie la sentence. Le juge consulte la fin de l'Évangile et finalement obtempère avec l'opinion de R. Gamaliel selon laquelle le fils a la priorité sur la fille dans une affaire d'héritage. Il convient de remarquer que le passage désire montrer que le juge préféra l'objet de R. Gamaliel à celui de sa sœur Imma Shalom. Ainsi, après avoir statué en faveur de Imma Shalom, il donne la préséance à $\mathrm{R}$. Gamaliel. D'où la fin chargée d'ironie du passage exprimant les propos de celle-ci : "Que ta lumière brille comme une lampe » et de R. Gamaliel : «L'âne est venu et a donné un coup de pied à la lampe ». On comprendra que l'objectif est de montrer que le jugement s'effectue en fonction de la valeur de l'objet de corruption et que l'on ne peut aucunement se fier à ce juge et à ce qu'il représente. On comprendra surtout que la consultation de l'Évangile n'est pas considérée comme une source fiable et fondatrice; elle n'aura servi qu'à modifier le jugement en fonction de l'objet de corruption. C'est donc d'une part le personnage, et d'autre part son texte, qui sont tournés en dérision.

Il convient de s'attarder quelque peu sur ce propos du juge : « Je ne suis venu ni pour retrancher à la loi de Moïse, ni pour ajouter à la loi de Moïse ». Ce verset qui est attesté dans les manuscrits ainsi que dans les éditions primitives sous la forme : « Je ne suis pas venu pour retrancher à la loi de Moïse, mais אלא pour ajouter à la loi de Moïse », est souvent perçu comme une retranscription de Mt 5, 17 : « N'allez pas croire que je sois venu abroger la loi ou les prophètes : je ne suis pas venu abroger mais accomplir ». Nous reviendrons ultérieurement sur l'analyse de ce verset dans le contexte de notre passage, cependant on peut déjà constater qu'une grande similitude syntaxique et terminologique apparaît dans les deux citations. La citation du verset de l'Évangile selon Matthieu qui sert de support scripturaire au juge pour statuer sur le cas exposé par Imma Shalom et R. Gamaliel (« J'ai consulté la fin de l'Évangile et il y est écrit (...) ), permet d'affirmer son identité judéo-chrétienne. Bien 
entendu, cette assertion se trouve renforcée par l'argument selon lequel l'exil de la terre d'Israël et l'interruption de la validité de la loi de Moïse ont entraîné le don de la nouvelle loi personnifiée par l'Évangile en remplacement de l'ancienne loi de Moïse ${ }^{53}$.

Il semblerait donc qu'il faille situer ce récit dans une perspective polémique entre les tenants de deux traditions certes encore au sein du judaïsme mais opposant des grilles et des modalités de lecture et d'appui scripturaires divergentes.

\section{Mt 5, 17 : Je ne suis pas venu abroger mais accomplir}

Dans le déroulement de l'histoire, après que R. Gamaliel apporte l'âne pour le corrompre, le juge modifie son jugement et cite littéralement la phrase suivante : " Je ne suis venu ni pour retrancher à la loi de Moïse, ni pour ajouter à la loi de Moïse ». À la lecture de cette maxime qui ressemble fort à une citation scripturaire, on ne peut que remarquer la ressemblance avec le verset évangélique connu de Mt 5, 17 : «Je ne suis pas venu abroger mais accomplir ». Ce verset s'imbrique dans un discours de Jésus communément intitulé

53. L'identité judéo-chrétienne du juge peut également être relevée à partir du vocable «philosophe » mentionné dans le texte. Ce terme apparaît dans le Midrash sur Psaumes 10, dans le contexte suivant: «C'est ce que dit le verset: "Avec leur menu et leur gros bétail, ils iront se mettre à la recherche de Dieu, mais ne le trouveront pas. Il s'est retiré d'eux" (Os 5,6). Un philosophe a demandé à R. Gamaliel : "Vous espérez que Dieu vous sauve". Il lui répondit : "Oui”. Il ajouta : "Mais, n'est-il pas écrit : "Il s'est retiré d'eux", une belle-sœur (promise à son beau-frère), libérée par ses beaux-frères, peut-elle retrouver son mari? Il lui dit [R. Gamaliel] : "non". Il lui dit : [le philosophe] : "D'ici [on apprend], que Dieu ne reviendra pas vers vous". R. Gamaliel lui dit : "Qui se désiste (du lévirat), l'homme ou la femme?" Il lui dit : "La femme". Il lui dit : "Dieu s'est retiré mais nous, nous ne nous sommes pas retirés de Lui, de la même façon, une femme (promise à son beau-frère), qui est libérée par ses beaux-frères n'a aucune obligation" ». Notons que dans le passage parallèle de Yebamoth 102b, c'est toujours R. Gamaliel qui est mentionné, mais qui débat avec un min. Ces deux textes sont donc deux versions d'un semblable épisode qui évoque une controverse entre R. Gamaliel et certainement un judéo-chrétien qui cherche à montrer que Dieu a abandonné le peuple juif. Il n'est pas sans importance que ce soit justement R. Gamaliel qui soit mentionné dans ce passage. Ce dernier semble en effet, avoir entretenu de nombreuses polémiques avec des judéo-chrétiens. Voir également H. Graetz, «Gnosticisme et judaïsme » dans La construction de l'histoire juive, Paris, 1992, p. 108 note 18, qui écrit : « Les chrétiens intelligents des premiers siècles aimaient mener une existence philosophique, c'est-à-dire stoïcienne, porter le pallium philosophorum et se nommer eux-mêmes des philosophes ». 
«Le sermon sur la montagne ». Il est souvent perçu et interprété par l'exégèse chrétienne comme étant l'accomplissement de la loi juive.

Il parait important de s'intéresser à certains aspects que recèle ce verset et à sa contextualisation dans le passage talmudique étudié. Il est évident que la démarche de Imma Shalom et de R. Gamaliel consiste à ridiculiser le juge judéo-chrétien et les textes sur lesquels il s'appuie. De la sorte, le fait qu'il consulte l'Évangile à deux reprises et qu'il en extraie à chaque fois des propos contradictoires est significatif. Bien entendu, son caractère corruptible ne fait que compléter l'image qu'on veut en donner.

La question de l'authenticité de ce passage ne fait pas l'unanimité parmi les critiques. En effet, la question de la diffusion des Évangiles, ou plus exactement de certains fragments, doit être posée concernant le $\mathrm{II}^{\mathrm{e}}$ siècle, époque des intervenants de ce passage. On peut, à ce titre, conjecturer que si toutefois le juge eut sous les yeux un quelconque support textuel utilisé dans les groupes judéochrétiens, ce n'était pas la forme des Évangiles tels qu'ils nous sont connus aujourd'hui, mais plutôt des fragments, peut-être apparents à la source $Q$ (Quelle).

K. G. Kuhn a suggéré de dater ce passage au $\mathrm{III}^{\mathrm{e}}$ siècle, bien qu'Imma Shalom et R. Gamaliel aient tous deux vécu au II siècle. Les noms de ces deux personnages n'apparaîtraient que pour conférer à l'histoire une certaine véracité. Il s'agirait néanmoins d'une reconstitution tardive, d'époque amoraïte, la tendance étant alors de critiquer le christianisme évoluant en dehors du judaïsme ${ }^{54}$.

En ce qui concerne la mention du verset de Mt 5, 17, dans le passage de Sabbath 116a-b, les critiques divergent une fois encore sur le fait de savoir s'il s'agit bien de ce verset qui est cité dans le Talmud $^{55}$.

54. Cf. K. G. Kuhn, « Giljonim und sifre minim », dans Judentum Urchristentum Kirche. Festschrift für Joachim Jeremias, Berlin, 1960, p. 53-57.

55. Voir à titre d'exemple: G. Dalman, Jesus Christ in the Talmud, Midrash, Zohar and the Liturgy of the Synagogue, New York, 1893, p. 69-70, qui estime que ce n'est pas d'une ancienne version des Évangiles que le juge a extrait cette citation, mais plutôt de sa mémoire défaillante; J. Maier, Jüdische Auseinandersetzung mit dem Christentum in der Antike, Darmstadt, 1982, p. 78-93, estime qu'on ne peut absolument pas retrouver le verset de Matthieu dans les propos du juge. Notons toutefois que la plupart des critiques s'entendent pour accepter ce passage comme historique; on peut citer à titre d'exemple R. T. Herford, Christianity in Talmud and Midrash, p. 147, qui est un des premiers chercheurs à avoir considéré ce texte ainsi. 
Certains critiques ont proposé de voir dans les propos de Imma Shalom et de R. Gamaliel des allusions satiriques à des versets matthéens. Il s'agit particulièrement des deux proverbes exprimés à la fin du passage Que ta lumière brille comme une lampe et L'ane est venu et a donné un coup de pied à la lampe. Les termes «lampe» et « âne » ne semblent en effet ne pas être anodins mais au contraire chargés d'un sens allusif.

M. Güdemann a considéré que ces deux expressions étaient à lire en corrélation avec Mt 5, 14-17. Selon ce critique, il ne fait aucun doute que des parallèles sont à déceler entre ces deux textes. Avant de reprendre les développements énoncés par l'auteur, il convient d'abord de citer les versets dans leur intégralité.

Mt 5, 15 : «On n'allume pas une lampe ( $\lambda$ úxvov : lukhnon) pour

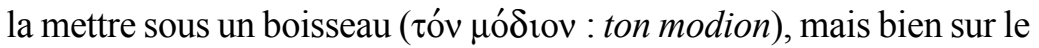
lampadaire, où elle brille pour tous ceux qui sont dans la maison ».

Mt 5, 16: «C'est ainsi que votre lumière doit briller devant les

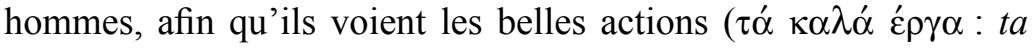
kala erga) que vous faites et qu'ils louent votre Père qui est dans les cieux $»$.

Selon M. Güdemann, le terme grec $\mu$ ó $\delta$ เov (modion traduit en français par «boisseau ») au verset 15 , correspondrait au vocable hébraïque חומר (homer). Ce terme de homer, désignant une ancienne mesure de capacité, serait rendu en grec par modion et traduit par « boisseau » qui définit également une ancienne mesure de capacité d'environ un décalitre. Enfin, R. Gamaliel aurait employé le terme araméen חמרא (hamra) qui se retrouve en hébreu sous la forme חמור (hamor) et désigne l'âne ${ }^{56}$. Ainsi, ce terme désignerait le boisseau et permettrait une relecture totale du verset matthéen sous la forme : «On n'allume pas une lampe pour la mettre sous un âne, mais bien sous le lampadaire, où elle brille pour tous ceux qui sont dans la maison ». Lecture qui serait en parfaite harmonie avec le propos de R. Gamaliel : «L'âne (hamra/homer) est venu et a mis un coup de pied à la lampe ».

Si cette suggestion est retenue, nous serions donc en présence de deux expressions talmudiques dans lesquelles des termes dissi-

56. Cf. M. Güdemann, Religionsgeschichtliche Studien, Vienne, 1876, p. 8085. Cette analyse est reprise par R. T. Herford, Christianity in Talmud and Midrash, p. $151-152$. 
muleraient des allusions à des versets de l'Évangile selon Matthieu. Cela au travers de la représentation métaphorique entre le boisseau et l'âne, tous deux pouvant être relatifs à une ancienne mesure de capacité. Cette suggestion permet au moins trois importantes remarques :

1. Au début du II $^{\mathrm{e}}$ siècle, R. Gamaliel et Imma Shalom avaient connaissance de certains éléments des versets 15 et 16 de l'Évangile selon Matthieu.

2. Ils se sont servi des mots contenus dans ces versets afin de tourner en dérision les idées qu'ils émettent.

3. R. Gamaliel a sciemment modifié le mot homer en hamor afin de mettre en évidence le caractère vain des propos évangéliques et l'importance que leur conféraient les judéo-chrétiens.

Cette dernière observation peut être renforcée par le langage araméen du passage de Sabbath 116a-b qui laisse supposer une rédaction tardive. Il est ainsi plus aisé d'estimer que les versets de Matthieu aient été connus. Cette démarche prend tout son sens à la lecture du verset 16; la lumière qui doit briller devant les hommes est totalement morte si elle est renversée par l'âne, on ne peut donc pas glorifier le « Père qui est aux cieux ». De la sorte, le discrédit est jeté sur les croyances judéo-chrétiennes et sur la messianité de Jésus ${ }^{57}$. On peut, à ce propos, rappeler la phrase de Jésus en Mt 5, 14 : «Vous êtes la lumière du monde », qui reprend l'idée de lumière en tant que dévoilement divin rédempteur. Il y a là un retraitement de ce concept tel qu'il figure dans la Bible et chez les prophètes. En effet, en Ps 27, 1, Dieu est lumière, alors qu'en Ps 119,105 , c'est la Torah qui est lumière, et le peuple d'Israël, qui accomplit et enseigne la Torah, est défini comme la « lumière des nations » d'après la prophétie d'Isaïe $(42,6 ; 49,6)$. Cette prophétie a été relue et appliquée à Jésus non seulement par les Évangiles en général $(\operatorname{Lc} 2,32 ;$ Jn 8, 12) mais aussi par Matthieu en particulier (voir 4, 16, « le peuple qui se demeurait dans les ténèbres a vu une grande lumière »). Il s'ensuit donc que tous les disciples en tant que participants à la vie du Messie sont « lumière du monde ». En effet, il n'est pas mentionné « vous êtes des lumières » mais « vous êtes

57. Voir B. L. Visotzky, « Overturning the Lamp », dans Journal of Jewish Studies 38 (1987), p. 72-80, qui reprend l'analyse de cette question et considère que dans les années $90 \mathrm{du} \mathrm{I}^{\mathrm{er}}$ siècle, les versets de Matthieu avaient plus ou moins une forme identique à celle qu'ils ont aujourd'hui (p. 79 note 22). 
lumière », considérés comme formant le corps du Messie qui est la lumière du monde ${ }^{58}$.

De tous les mots de l'Évangile selon Matthieu sur le thème de la lumière, « lampe » est le seul qui se trouve déjà chez Marc (Mc 4, 21), il est à comprendre comme quelque chose de mis en marche, dont on peut dire qu' "il vient », n'étant pas encore allumé. Cela permet donc de dire que, dans l'Évangile selon Matthieu, le terme «lampe » est interprété dans un sens messianique ${ }^{59}$. Dans un sens plus pratique, il convient de rappeler que le boisseau est un récipient dont l'usage était, entre autres, d'éteindre une lampe.

C'était vraisemblablement de façon délibérée que R. Gamaliel et Imma Shalom ont employé les termes « lampe » et « âne » dans ce proverbe, l'objectif étant de montrer la vacuité du message des versets matthéens et de les tourner en dérision.

\section{L'origine hébraïco-araméenne de l'Évangile selon Matthieu}

L'origine hébraïque de l'Évangile selon Matthieu appartient à une longue tradition patristique. $\mathrm{Au} \mathrm{XVI}^{\mathrm{e}}$ siècle deux versions hébraïques de cet évangile sont éditées : en 1537 par Sebastian Münster et en 1555 par Jean du Tillet, évêque de Brieux. Cette seconde version fut traduite en latin par un hébraïste du nom de Jean Mercier ${ }^{60}$. C'est d'ailleurs dans le contexte d'un regain d'inté-

58. Cette glose est d'origine byzantine et se trouve citée par A. H. McNeile, The Gospel According to St. Matthew, Grands Rapids, 1980², p. 56; voir également sur ce verset S. T. Lachs, "Studies in the Semitic Background to the Gospel of Mattew », dans Jewish Quarterly Review 67 (1977), p. 199-202 qui reprend brièvement ce dossier et propose d'intéressantes remarques philologiques.

59. On doit rappeler que l'inscription la plus courante sur les lampes byzantines était : «La lumière du Messie brille pour vous » (Voir A. Mello, Évangile selon Saint Matthieu, p. 118 note 1).

60. La version de Sebastian Münster est parue en 1537 à Basiliae sous le titre Evangelium secondum Matthaeum in lingua Hebraica, cum versione latina atque succinctis annotationibus. La version de Jean du Tillet est parue à Paris en 1555 sous le titre Evangelium Matthaei ex Hebraeo fideliter redditum. Elle fut traduite en anglais par H. J. Schonfield sous le titre An Old Hebrew Text of St. Matthew's Gospel et publiée à Édimbourg en 1927. Voir l'étude consacrée à cette version de G. Howard, "The Textual Nature of an Old Hebrew Version of Matthew ", dans Journal of Biblical Literature 105 (1986), p. 49-63. Dans cette étude, Howard parvient à la conclusion que la version de Jean du Tillet n'est pas la version du Matthieu hébreu à laquelle font mention les Pères de l'Église. 
rêt pour la langue hébraïque propre au $\mathrm{XVI}^{\mathrm{e}}$ siècle, que les versions traduites ont vu le jour ${ }^{61}$.

Il convient de souligner que la communauté de l'évangéliste Matthieu semble appartenir historiquement au prolongement de celle provenant de la « source des logia ». Selon U. Luz, la communauté matthéenne est judéo-chrétienne, née de l'œuvre des disciples et messagers de Jésus, ceux-là même qui étaient dépositaires de la tradition de la « source des logia » et qui s'établirent en Syrie postérieurement à l'insurrection juive de l'année 66 .

Selon ce critique, l'Évangile selon Matthieu proviendrait d'une communauté judéo-chrétienne qui, à l'issue de la catastrophe de l'année 70, se serait ouverte à la "Grande Église » pagano-chrétienne, tout en préservant son identité initiale ${ }^{62}$.

Selon A. J. Saldarini, la communauté matthéenne et son porteparole, l'auteur de l'Évangile de Matthieu, sont des juifs qui ont cru en Jésus en tant que Messie et Fils de Dieu. Cette communauté matthéenne représenterait une minorité précaire se considérant comme juive, et encore identifiée à la communauté juive par les autres. En dépit de ses violentes confrontations avec certains responsables de la communauté juive, le groupe matthéen est encore pleinement juif. En outre, l'auteur de l'Évangile de Matthieu se perçoit comme un juif détenteur de la véritable interprétation de la Torah, fidèle à la volonté divine révélée par Jésus qu'il déclare être le Messie et le Fils de Dieu. Il tente de promouvoir son interprétation du judaïsme en lieu et place de celle des autres responsables juifs, de ceux qui mettent en œuvre le judaïsme rabbinique. L'évangéliste Matthieu maintient Israël à sa place parmi les nations bien que son objectif soit d'ouvrir les frontières d'Israël afin d'accueillir les païens croyant en la Torah et observant certaines pratiques juives. En bref, il est possible de dire que pour Saldarini, l’Évangile

61. Cf. W. Horbury, "The Hebrew Matthew and Hebrew Study», dans W. Horbury (ed.), Hebrew Study from Ezra to Ben-Yehuda, Edimbourg, 1999, p. 122-131.

62. Voir U. Luz, «L'évangéliste Matthieu: un judéo-chrétien à la croisée des chemins. Réflexions sur le plan narratif du premier évangile», dans D. Marguerat; J. Zumstein (Éds), La mémoire et le temps, Genève, 1991, p. 7792. Il est des plus souhaitables de considérer les propos de S. C. Mimouni, « Le cas de l'Évangile selon Matthieu, un Évangile à la croisée des chemins », dans Le judéo-christianisme ancien. Essais historiques, Paris, 1998, p. 108-110, sur la présentation de ce travail. 
selon Matthieu est à situer parmi la fragmentation juive propre au $\mathrm{I}^{\mathrm{er}}$ siècle. ${ }^{63}$

Cette approche qui peut être définie comme une forme de christianisme juif est reconsidérée par Donald Hagner qui préfère parler de forme juive de christianisme. Selon Hagner, la pratique ritualiste présente dans Matthieu prédomine sur son identité bien que cette dernière soit également constituée de la croyance en la messianité de Jésus. À la différence de Saldarini qui définit la communauté matthéenne comme une secte, Hagner parle plutôt en termes de culte, suivant en cela la terminologie proposée par Petri Luomanen. On notera que ces critiques considèrent l'Évangile selon Matthieu en tant que religious innovation et ajoutent qu'il a grandement contribué au phénomène de parting of the ways, c'est-à-dire de la séparation entre le groupe chrétien et le judaïsme. Selon Hagner, les enseignements de Matthieu sont à percevoir comme une césure avec le judaïsme à cause notamment de la croyance messianique, de l'annonce eschatologique, de la rémission des péchés accordée à un messie crucifié tel un criminel, de la focalisation sur le personnage de Jésus en tant qu'interprète autorisé de la Loi, de l'immixtion des païens au sein de la nouvelle communauté ${ }^{64}$. Notons, avec Warren Carter, que la question de la part plutôt juive ou plutôt chrétienne de l'Évangile selon Matthieu est loin d'être consensuelle et doit peutêtre être repensée en fonction d'autres réalités, comme par exemple le fonctionnement interne du pouvoir romain ${ }^{65}$.

63. Cf. A. J. Saldarini, Matthews's Christian-Jewish Community, ChicagoLondres, 1994. Voir les critiques formulées contre les thèses de Saldarini et notamment sur la question de la christologie par D. Hare, « How Jewish is the Gospel of Matthew? », dans Catholic Biblical Quarterly 62 (2000), p. 264-277.

64. Cf. P. Luomanen, "The Sociology of Sectarianism in Matthew: Modeling the Genesis on Early Jewish and Christian Communities », dans I. Dunderberg; C. Tuckett; K. Syreeni (eds.), Fair Play. Diversity and Conflicts in Early Christianity : Essays in Honnour of Heikki Räisänen, Leyde, 2002, p. 107130; D. Hagner, « Matthew : Apostate, Reformer, Revolutionary », dans New Testament Studies 49 (2003), p. 193-209; D. Hagner, « Matthew : Christian Judaism or Jewish Christianity », dans S. McKnight; G. Osborne (eds.), The Face of New Testament Studies : A Survey of Recent Research, Gand Rapids, 2004, p. 263282.

65. Cf. récemment W. Carter, «Matthew's : Jewish Christianity, Christian Judaism, or Neither », dans M. Jackson-McCabe (ed.), Jewish Christianity Reconsidered, Minneapolis, 2007, p. 155-179 qui propose un article de synthèse sur les thèses de Saldarini et de Hagner et fait le point sur les points les plus significatifs qui les constituent. 
On soulignera - sans toutefois s'attarder sur ce dossier - que les catégories proposées par ces critiques restent d'une certaine façon totalement artificielles. En effet, elles peuvent être pertinentes d'un point de vue conceptuel mais le sont moins dans une perspective purement historienne. Il est en effet fondamental avant toute démarche taxinomique de bien situer historiquement les problématiques : les notions de judaïsme et de christianisme sont, à l'époque où Matthieu rédige son évangile, loin d'être claires et uniformes. Le judaïsme rabbinique des Sages du Talmud et le christianisme orthodoxe ne sont pas encore constitués à une époque aussi reculée que les années 80/90 du $\mathrm{I}^{\text {er }}$ siècle de l'ère chrétienne ${ }^{66}$.

En ce qui concerne l'origine de cet Évangile, Eusèbe de Césarée, citant Papias, consigne que Matthieu aurait rassemblé les « logia » de Jésus en langue hébraïque, chacun alors les traduisant et les interprétant ${ }^{67}$. Il s'agirait donc d'un Évangile de langue araméenne ou même hébraïque ${ }^{68}$. Ce témoignage se trouve renforcé par Irénée

66. Cf. F. J. Murphy, «The Jewishness of Matthew : Another Look », dans A. J. Avery-Peck; D. Harrington; J. Neusner (eds.), When Judaism and Christianity Began. Essays in Memory of Anthony J. Saldarini, Leyde/Boston, 2004, v. II, p. 377 qui écrit : « Scholars have approached the text with analytical categories based on later periods, when christianity and Judaism had become two distinct religions. But when Matthew was written, the distinction was not clear ». Soulignons que nous envisageons de revenir à ce dossier dans une prochaine publication.

67. Voir Eusèbe de Césarée, Histoire écclésiastique, III, 24, 6; 39, 16 (Sources Chrétiennes, Vol. I, p. 130, 157); Irénée de Lyon, Contre les hérésies, Chap. III, 1, 1 (trad. A. Rousseau, Paris, 1991), p. 277. Voir également les travaux sur ce dossier: J. Kürzinger, «Das Papiaszeugnis und die Erstgestalt des Matthäusevangeliums », dans Biblische Zeitschrift4(1960), p. 19-38; J. Kürzinger, «Irenäus und sein Zeugnis zur Sprache des Matthäusevangeliums », dans New Testament Studies 10 (1963), p. 108-115; J. Kürzinger, « Die Aussage des Papias von Hierapolis zur literarischen Form des Markusevangeliums », dans Biblische Zeitschrift 21 (1977), p. 245-264; J. Kürzinger, « Papias von Hierapolis : Zu Titel und rt Seines Werkes », dans Biblische Zeitschrift 23 (1979), p. 172-186 et enfin son ouvrage Papias von Hierapolis und die Evangelien des Neuen Testaments, Regensburg, 1983. La conclusion à laquelle parvient Kürzinger est que Papias se réfère seulement au style d'écriture de Matthieu et non au langage dans lequel il écrit. Il est cependant difficile d'être convaincu par cette approche. L'argumentaire de Kürzinger est basé sur l'hypothèse selon laquelle Papias utilisait une ancienne terminologie rhétorique, toutefois cela revient à lui attribuer plus d'érudition que ce qu'il devait réellement posséder. Eusèbe lui-même décrit d'ailleurs Papias comme un homme de peu d'intelligence (Histoire ecclésiastique III, 39, 13).

68. Cf. G. Howard, The Gospel of Matthew according to a Primitive Hebrew Text, Macon, 1987, p. 155-156 refuse l'argument selon lequel l'hébreu n'était plus utilisé en Palestine au temps de Jésus en mentionnant les Manuscrits de la mer 
de Lyon prétendant que Matthieu rédigea un Évangile de langue

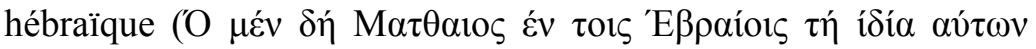
$\delta 1 \alpha \lambda \varepsilon ́ \kappa \tau \omega)$ durant son séjour chez les Hébreux. De plus, selon Irénée, cet Évangile est le premier des synoptiques.

Dans son Commentaire sur Matthieu Origène mentionne également l'existence d'un Évangile selon Matthieu en hébreu :

Comme je l'ai appris dans la tradition au sujet des quatre Évangiles qui sont aussi seuls incontestés dans l'Église de Dieu qui est sous le ciel, d'abord a été écrit celui qui est selon Matthieu, premièrement publicain, puis apôtre de Jésus-Christ : il l'a édicté pour les croyants

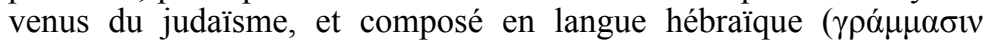
'E

Epiphane de Salamine (Panarion 29, 9, 4), précise que les nazoréens ne connaissent qu'un Évangile selon Matthieu écrit en hébreu :

Ils ont aussi l'Évangile selon Matthieu d'une manière très complète, en hébreu, car chez eux il est manifestement conservé en caractères hébraïques comme il était écrit à l'origine. Mais je ne sais pas s'ils en ont retiré aussi les généalogies depuis Abraham jusqu'au Christ.

A. F. J. Klijn a estimé qu'Epiphane n'a pas eu en main l'Évangile selon Matthieu en hébreu car il ignore s'il contient ou non les généalogies. De ce fait, il infère qu'Epiphane pourrait faire référence à un Évangile apocryphe ${ }^{70}$.

A. Pourkier, pour sa part, suggère qu'Epiphane fait réellement référence à l'Évangile canonique de Matthieu, notamment car Papias, Irénée, Origène et Eusèbe ont émis l'idée que sa langue originale était l'hébreu. Elle justifie le propos sur les généalogies par le fait qu'Epiphane, sachant que les ébionites ont retranché les généalogies de l'Évangile selon Matthieu ${ }^{71}$, se demande si les nazoréens en ont fait de même ${ }^{72}$.

Morte et le nombre important de textes hébraïques qu'ils comportent. En conséquence, Howard estime que le Matthieu originel a vraisemblablement pu être rédigé en hébreu et non en araméen.

69. Origène, Commentaire sur Matthieu I, cité par Eusèbe de Césarée, Histoire ecclésiastique, VI, 25, 4 (Sources Chrétiennes 41, Vol. II, p. 126).

70. Cf. A. F. J. Klijn, « Patristic Evidence for Jewish Christian and Aramaic Gospel Tradition », dans E. Best, R. Mc L. Wilson (Eds), Text and Interpretation. Studies in the New Testament presented to Matthew Black, Cambridge, 1979, p. 175.

71. Cf. Epiphane de Salamine, Panarion 30, 14, 3.

72. Cf. A. Pourkier, L'hérésiologie chez Epiphane de Salamine, Paris, 1992, p. 473. 
Quoi qu'il en soit, comme le fait remarquer S. C. Mimouni, la mention d'une origine hébraïque de l'Évangile selon Matthieu chez Epiphane de Salamine est une «information d'ordre historique à ne pas négliger, semble-t-il, dans le cadre du dossier de l'Évangile selon Matthieu en hébreu ${ }^{73}$.

A. F. J. Klijn considère qu'il était assez généralement reçu, parmi les auteurs chrétiens anciens, que l'évangile canonique de Matthieu avait été écrit en hébreu ou en araméen au sein d'une communauté judéo-chrétienne d'expression sémitique ${ }^{74}$. D'autres critiques tels W. D. Davies et D. Allison concluent à la fiabilité du témoignage de Papias quant à une origine juive du premier évangile, dans un milieu baigné dans la double culture, sémitique et hellénistique ${ }^{75}$. Toutefois, l'Antiquité ignorait déjà quel fut le traducteur en grec de cet écrit. Il semblerait donc que l'Évangile selon Matthieu soit une retranscription, voire une reproduction, comportant des ajouts, de la source hébraïque ou araméenne originale.

Il est donc possible de conclure que l'Évangile selon Matthieu se fonde sur un recueil primitif de langue hébraïque ou araméenne, lui-même certainement constitué à partir de la " source de logia ", qui fut plus tard traduit en $\operatorname{grec}^{76}$. F. Blanchetière estime que la rédaction de l'Évangile de Matthieu est le fruit d'une succession d'étapes qui ainsi ferait droit aux traditions rapportées notamment par Papias et Irénée. De la sorte, une première rédaction en araméen pour les besoins de la communauté matthéenne encore très centrée sur le monde juif aurait été suivie d'une refonte, d'une réélabora-

73. Cf. S. C. Mimouni, « Qui sont les Jesséens dans la notice 29 du Panarion d'Epiphane de Salamine? », dans Novum Testamentum 43 (2001), p. 292.

74. Cf. A. F. J. Klijn, Jewish-Christian Gospel Tradition. Supplements to Vigiliae Christianae 17, Leyde, 1992, p. 26. Notons également que Jérome fait référence à un Évangile selon Matthieu en hébreu en mentionnant de même un Évangile des Hébreux. Il est en outre bien malaisé de distinguer ces deux corpus chez Jérome. Voir à ce titre G. Howard, The Gospel of Matthew according to a Primitive Hebrew Text, p. 158-159.

75. W. D. Davies; D. Allison, A Critical and Exegetical Commentary of the Gospel according to Matthew, Edimbourg, 1988-1996, Vol. I, p. 7-58.

76. Voir R. M. Grant, Introduction historique au Nouveau Testament, Paris, 1969, p. 99-100; C. H. Schelkle, Introduction au Nouveau Testament, Paris, 1965, p. 49-50. H. Conzelmann; A. Lindemann, Guide pour l'étude du Nouveau Testament, Genève, 1999, p. 356-359, semblent nier l'origine hébraïque de l'Évangile selon Matthieu, de même qu'ils voient en Matthieu un Juif de naissance connaisseur de la Loi, plutôt qu'un judéo-chrétien scrupuleux envers l'observance rituelle. 
tion, d'une réécriture en grec dans un milieu plus ouvert à des tendances universalistes ${ }^{77}$.

Un passage du Contre les Hérésies $(1,26,2)$ d'Irénée de Lyon peut-être considéré comme une pièce supplémentaire au dossier de l'Évangile de Matthieu. Dans ce passage, Irénée consacre une notice aux ébionites en ces termes :

Ceux qu'on appelle ébionites admettent que le monde a été fait par le vrai Dieu, mais, pour ce qui concerne le Seigneur, ils professent les mêmes opinions que Cérinthe et Carpocrate. Ils n'utilisent que l'Évangile selon Matthieu, rejettent l'apôtre Paul qu'ils accusent d'apostasie à l'égard de la Loi. Ils s'appliquent à commenter les prophéties avec une minutie excessive (curiosius). Ils pratiquent la circoncision et persévèrent dans les coutumes légales et dans les pratiques juives, au point d'aller jusqu'à adorer Jérusalem, comme étant la maison de Dieu. ${ }^{78}$

Soulignons d'emblée que cette notice a une importance particulière : c'est le plus ancien des témoignages hérésiologiques conservés et il a exercé une forte influence sur la tradition subséquente. Ce passage, important à plus d'un titre, fait mention d'un élément crucial pour notre dossier, à savoir l'emploi exclusif de l'Évangile de Matthieu. Si la valeur de cette information est fiable historiquement, nous sommes ici avec un texte reflétant une des caractéristiques du judéo-christianisme au II $^{\mathrm{e}}$ siècle.

\section{Conclusion}

Il a été question dans cette contribution de montrer que les tannaïm avaient une certaine connaissance de versets issus de l'Évangile selon Matthieu dès le II $^{\mathrm{e}}$ siècle. Certes, cette connaissance certainement parcellaire se manifeste au travers d'une volonté de la part des Sages du Talmud de tourner en dérision la nature et le contenu des passages évangéliques. En considérant l'origine hébraïque de cet Évangile, il devient possible de conjecturer que les Sages ont considéré ces textes et se sont exprimés à leur endroit. Si cette hypothèse est retenue, nous serions en présence d'un phénomène extrêmement important du point de vue historique : la version origi-

77. Cf. F. Blanchetière, Enquête sur les racines juives du mouvement chrétien (30-135), Paris, 2001, p. 101-102.

78. Cf. Irénée de Lyon, Contre les hérésies, I, 26, 2, p. 117. 
nelle de l'Évangile selon Matthieu de laquelle on pourrait obtenir quelques bribes grâce au corpus talmudique. Il est vrai que longtemps la recherche historique n'a pas considéré avec pertinence les attestations patristiques sur l'origine hébraïque de l'Évangile selon Matthieu. Il semblerait toutefois que l'on doive reprendre en main ce dossier avec la plus grande attention, et ce pour au moins deux raisons : d'une part, car les témoignages historiques sur la communauté matthéenne confirment son aspect judéo-chrétien; d'autre part, car les textes talmudiques attestent des corrélations linguistiques et philologiques qui ne peuvent être anodines.

Nous serions ainsi en présence de deux phénomènes capitaux : - l'origine hébraïque ou araméenne de l'Évangile selon Matthieu dont certains éléments pourraient être décelés de façon paradoxale dans la littérature talmudique; - la connaissance des Sages du Talmud, à une époque aussi reculée que le $\mathrm{II}^{\mathrm{e}}$ siècle, de fragments de cet Écrit, qui deviendra avec le temps un des textes fondateurs de toute la chrétienté.

Dans notre démarche nous souhaiterions souligner une originalité : celle qui consiste à prendre comme support textuel la littérature talmudique afin de remonter jusqu'à une éventuelle origine hébraïque de l'Évangile selon Matthieu. Bien entendu, cette approche n'est pertinente que si l'on postule une véracité historique des propos des Pères de l'Église sur cet évangile et sur son langage originel. 
